\title{
Varicella Zoster Virus Vasculopathy in Adult: A Rare Case Report
}

\author{
Saurabh Saigal*, Ratender K Singh, Garima Kapoor and Arvind K Baronia
}

Department of Critical Care Medicine, Sanjay Gandhi Post Graduate Institute of Medical Sciences, Lucknow, UP, India-226014

\begin{abstract}
Varicella-zoster virus (VZV) is the only virus in human beings that has been shown to replicate in arteries and produce vasculopathy. Varicella-zoster virus (VZV) vasculopathy is a serious entity, producing considerable neurologic deficit and sometimes death. VZV produces either large vessel or small vessel vasculopathy in the CNS. Immunocompetent patients commonly have large-vessel disease whereas immunocompromised usually develop small-vessel vasculopathy. Patients presenting with neurological deficits that are suggestive of a vasculopathy, even in the absence of rash or CSF pleocytosis, should undergo virological testing for VZV. Here we present a rare case of varicella zoster virus induced vasculopathy.
\end{abstract}

Keywords: Varicella zoster virus; Vasculopathy

Abbreviations: Varicella zoster virus (VZV); Mean arterial pressure (MAP); Total leucocyte count (TLC); Liver function test (LFT); Aspartate Amino Transferase (AST); Alanine Amino Transferase (ALT); Human Immunodeficiency Virus (HIV); Ultrasonography (USG); Glasgow Coma Scale (GCS); Computed Tomography (CT); Cerebrospinal fluid (CSF); Human Immunodeficiency Virus (HIV); Magnetic resonance imaging (MRI); Magnetic resonance angiography (MRA)

\section{Introduction}

Primary infection with varicella-zoster virus (VZV), a neurotropic alpha herpes virus produces 4 million cases of chickenpox annually [1]. After chickenpox, VZV becomes latent in cranial nerve, dorsal root and autonomic nervous system ganglia along the entire neuraxis. Virus reactivation, most commonly seen in elderly and immunocompromised individuals, produces zoster (shingles). Less often, VZV reactivates from ganglia and spreads to arteries of the brain and spinal cord producing vasculopathy [1].

\section{Case Report}

A 42 years/male patient with past history of diabetes induced nephropathy since 2 years, presented with lower respiratory tract infection for 15 days and oliguria since 10 days. Owing to his declining renal function and progressively increasing sepsis he was shifted to our ICU.

On admission he was tachycardic, tachypnoeic and had mean arterial pressure (MAP) $<65 \mathrm{mmHg}$ with cool extremities. On auscultation, bilateral wheeze was present and bilateral hilar congestion was evident on chest X-ray. Arterial blood gas analysis showed primary severe metabolic acidosis with compensatory respiratory alkalosis. Since he was in refractory septic shock with oliguria, vasopressors were started and slow low efficiency dialysis was initiated. His hemoglobin was $7.8 \mathrm{gm} / \mathrm{L}, \mathrm{TLC}=23,300 / \mathrm{mm}^{3}$, platelets $=63,000 / \mathrm{mm}^{3}$, LFT-serum bilirubin $=0.9 \mathrm{mg} / \mathrm{dl}, \mathrm{AST} / \mathrm{ALT}=51 / 29 \mathrm{IU} / \mathrm{L}$, serum albumin $=2.8 \mathrm{mg} / \mathrm{dl}$, serum creatinine $=7.8 \mathrm{mg} / \mathrm{dl}$. Malaria, typhoid, leptospira, dengue and HIV serology were all negative. USG abdomen revealed bilateral small kidneys with poor cortico-medullary differentiation. Left ventricular hypertrophy was the only positive finding on echocardiography and his lower limb Doppler study was normal.

His clinical condition started improving over the next two weeks, leucocytosis decreased, hemodynamics stabilized and urine output improved with alternate day dialysis. He however developed recent onset high grade fever with temperatures up to 40 degree Celsius. Two days after fever, vesicular eruptions were noticed on trunk, face and extremities. Since these vesicles appeared in crops, were bilaterally present (not specific for any dermatome) and centripetal in location, provisional diagnosis of chicken pox was made which was later further validated by visualizing inclusion bodies in Tzanck smear from vesicles. Also anti VZV IgM antibody was detected in the sera after 5 days of fever.

After renal dose modification, intravenous Acyclovir $800 \mathrm{mg}$ twice daily was started. However, patient's sensorium continued to deteriorate. He was withdrawing to painful stimulus with no focal neurological deficit (GCS was $\mathrm{E}_{2} \mathrm{~V}_{\mathrm{T}} \mathrm{M}_{4}$ ); pupils bilaterally equal and reacting to light, plantars mute, deep tendon reflexes exaggerated and in order to protect his airways he required tracheal intubation followed by mechanical ventilation. Plain CT scan of head was normal, CSF examination revealed normal pressure, clear fluid, 600 cells, $90 \%$ polymorph, protein $=97 \mathrm{mg} / \mathrm{dl}$, sugars $=28 \mathrm{mg} / \mathrm{dl}$. India ink preparation for Cryptococcus spp. and adenosine deaminase (ADA), ZN staining, PCR were all negative for Mycobacterium tuberculosis, Anti-VZV antibody in CSF was positive. Magnetic resonance imaging (MRI) (Figure 1) showed multiple discrete hyper intensities in bilateral deep and periventricular white matter suggestive of multifocal vasculopathy. His magnetic resonance angiography (MRA) showed narrow constrictions in region of internal carotid artery also suggestive of vasculopathy. Diagnosis of VZV vasculopathy was made. Intravenous methylprednisolone (1 gm/day) was started for 3 days followed by once daily oral dose of Prednisolone of $60 \mathrm{mg}$ for next 7 days with no taper. Intravenous Acyclovir was continued for 21 days. In view of prolonged ventilation, tracheostomy was done. Over the next 10 days, his sensorium started improving and he started localizing pain. At present, he is still in our ICU conscious, oriented, obeying simple commands, recovering from renal failure and is being weaned off the ventilator.

${ }^{*}$ Corresponding author: Saigal Saurabh, Senior resident (CCM), Department of Critical Care Medicine, Sanjay Gandhi Postgraduate Institute of Medical Sciences Lucknow, India-226014, Tel: +91 522 2494540; Fax: +91 522 2668017, E-mail: saurabhda_2006@rediffmail.com

Received November 09, 2011; Accepted January 21, 2012; Published January 28, 2012

Citation: Saigal S, Singh RK, Kapoor G, Baronia AK (2012) Varicella Zoster Virus Vasculopathy in Adult: A Rare Case Report. J Clinic Case Reports 2:110. doi:10.4172/2165-7920.1000110

Copyright: ( 2012 Saigal S, et al. This is an open-access article distributed under the terms of the Creative Commons Attribution License, which permits unrestricted use, distribution, and reproduction in any medium, provided the original author and source are credited. 


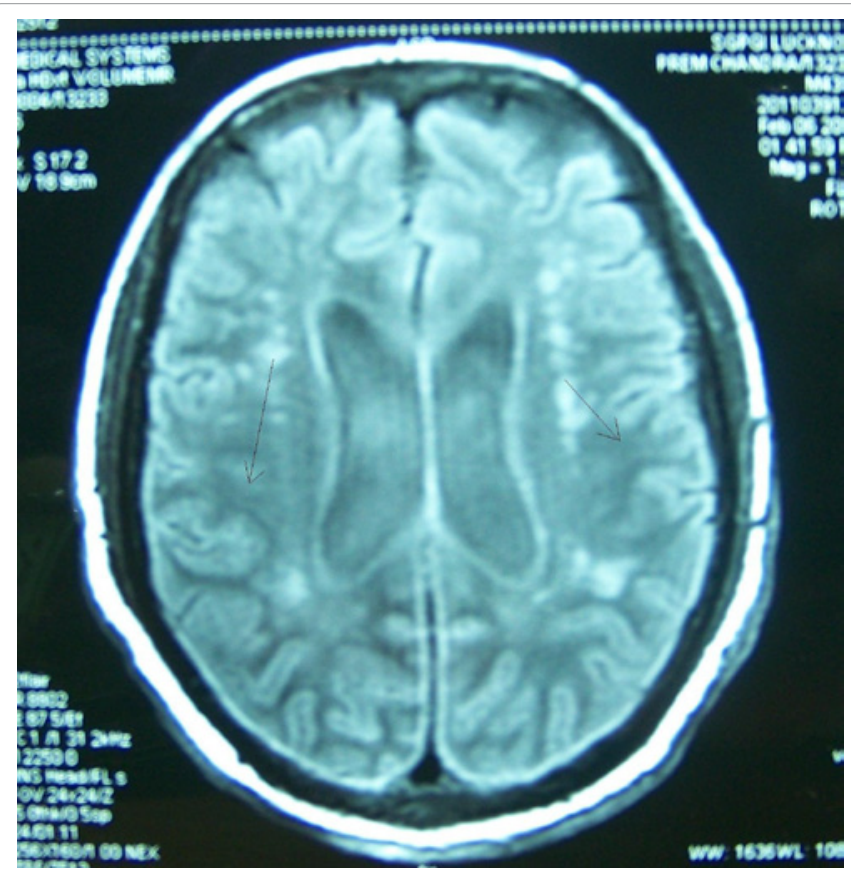

Figure 1: MRI findings: Multiple discrete hyperintensities in bilateral deep and periventricular white matter

\section{Discussion}

VZV is the only virus in human beings that has been shown to replicate in arteries and produce vasculopathy [2]. VZV infection in the CNS produces either unifocal (large vessel) or multifocal (small vessel) vasculopathy. In multifocal vasculopathy, smaller infarcts in deep white and gray matter reflect transaxonal transport of VZV from trigeminal or cervical afferent fibers to vessels of the posterior circulation [3]. The infection may produce a combination of headache, fever, mental status changes and multifocal deficit; evident on neurological examination and MRI brain. In unifocal vasculopathy, large-vessel infarcts can result in acute focal neurological deficit, due to transaxonal spread of VZV in anterior circulation [3]. Thus the clinical and MRI findings in our case were suggestive of multifocal vasculopathy.

Compared to zoster and post herpetic neuralgia, VZV vasculopathy is quite serious, producing considerable neurological deficit [1]. Immunocompromised patients, like ours, usually develop small-vessel vasculopathy whereas immunocompetent patients commonly have large-vessel disease [3]. However, both sized vessels may be involved in some patients. Often the neurological disease may occur months after the zoster and can sometimes occur even without a rash [1].

CSF virological analysis, MRI scanning and cerebral angiography are needed to confirm the diagnosis of this rare infective vasculopathy. VZV DNA may not always be present in CSF but the presence of anti-VZV antibody in CSF along with reduced serum/CSF ratios of VZV immunoglobulin G (IgG) compared to albumin or total IgG are confirmatory of the diagnosis [1].

In our patient, chicken pox was confirmed by microbiological analysis of vesicular fluid and also anti VZV IgM antibody was positive in the sera and CSF. Initial neutrophilic leucocytosis and low glucose levels as seen in our patient's CSF can be seen in early phase of Varicella infection. Patients with varicella may have secondary bacterial infections commonly due to Staphylococcus aureus and Streptococcus pyogenes, hence we empirically added ceftriaxone and vancomycin as cover for bacterial meningitis. In view of MRI findings suggestive of multifocal vasculopathy and rapidly deteriorating neurological status, we started pulse therapy of methylprednisolone followed by oral prednisolone. This along with intravenous acyclovir resulted in remarkable improvement in patient's neurological condition.

Once clinical features of patient suggest encephalitis, we usually think of HSV infection [4] and rule it out by CSF examination and MRI. But as VZV infection presenting as vasculopathy is also common and usually presents without rash in immunocompromised individuals; hence a high degree of suspicion is needed to reach the diagnosis otherwise the antiviral medications may be prematurely discontinued.

VZV vasculopathy is known to produce considerable neurological deficit [1]. Though mortality for varicella vasculopathy is $5 \%-10 \%$, but long term sequelae like seizure disorders, may be present in 10\%-20\% of survivors [5].

VZV is a neurotropic virus that infects CNS blood vessels of various calibers. The disease perse is not primarily encephalitis, but vasculopathy that develops secondary to VZV infection within large and small cerebral arteries leads to unifocal or multifocal infarction. Thus, patients presenting with neurological deficits that are possibly suggestive of a vasculopathy, even in the absence of rash or CSF pleocytosis, should undergo virological testing for VZV.

\section{Acknowledgement}

Department of Neurology, Sanjay Gandhi Postgraduate Institute of Medical Sciences, Lucknow.

\section{References}

1. Gilden DH, Mahalingam R, Cohrs RJ, Kleinschmidt-De Masters BK, Forghan $B(2002)$ The protean manifestations of varicella-zoster virus vasculopathy. $J$ Neuro Virol 8: 75-79.

2. Gilden DH, Cohrs RJ, Mahalingam R, Nagel MA (2009) Varicella zoste virus vasculopathies: diverse clinical manifestations, laboratory features, pathogenesis and treatment. Lancet Neurol 8: 731-740.

3. Gilden DH, Lipton HL, Wolf JS, Akenbrandt W, Smith JE, et al. (2002) Two patients with unusual forms of varicella-zoster virus vasculopathy. $N$ Engl $J$ Med 347: 1500-1503.

4. Huppatz C, Durrheim DN, Levi C, Dalton C, Williams D, et al. (2009) Etiology of encephalitis in Australia, 1990-2007. Emerg Infect Dis 15: $1359-1365$.

5. Gnann John W (2002) Varicella-Zoster Virus: Atypical Presentations and Unusual Complications. J Infect Dis 186: S91-S98. 